\title{
Using decision aids to help patients navigate the "grey zone" of medical decision-making
}

\section{Annette M. O'Connor}

$\infty \quad$ See related article page 1583

$\mathrm{M}$ any medical decisions exist in a "grey zone," where the best choice differs depending on how patients weigh the benefits and risks. A common example is the decision of whether to initiate a more aggressive treatment when simpler therapies fail to control moderate or severe symptoms, such as whether to choose hormone replacement therapy for symptoms of menopause; selective cyclooxygenase-2 inhibitors or joint replacement surgery for osteoarthritis; surgery for benign prostatic hypertrophy or back pain; or endometrial ablation or hysterectomy for benign uterine bleeding. Such decisions also commonly arise with issues involving genetic testing, reproductive choices (e.g., birth control, infertility options, vaginal birth after cesarean section), treatment of early cancer (e.g., mastectomy v. breast-conserving surgery; prostatectomy $\mathrm{v}$. radiation therapy), and the intensity of care at the beginning and end of life.

As discussed by Holbrook and coauthors in this issue,${ }^{1}$ the choice of anticoagulant therapy for atrial fibrillation is another "grey zone" decision for patients. Although warfarin is more effective than acetylsalicylic acid in preventing strokes, it brings with it a higher risk of major bleeds plus the inconvenience of having the international normalized ratio monitored and managed. Consequently, there is significant variation in how patients weigh the benefits and risks. ${ }^{2}$

Patient decision aids are adjuncts to counselling that prepare patients for discussions about treatment options with their physicians. They help patients to understand choices, outcomes and probabilities tailored to their clinical risk profile and to clarify which positive and negative consequences matter most to them. ${ }^{3}$ In the context of anticoagulation for atrial fibrillation, patient decision aids have been found to be superior to general education for increasing the proportion of patients whose international normalized ratios are managed in the appropriate range (absolute improvement of $19 \%$ at 3 months), although other strategies may be needed to main- tain long-term effects. ${ }^{4}$

With mounting evidence for the efficacy of patient decision aids (the Cochrane database currently lists 55 randomized controlled trials), the increasing number of available decision aids (currently more than 500 exist) and increasing access through the Internet (decision aids were accessed approximately 9 million times last year), issues about standards have been raised. ${ }^{5}$ Last year, an international collaboration of researchers, practitioners, patients and policy-makers endorsed the first set of standards for the content, development and evaluation of patient decision aids. ${ }^{6}$ However, issues regarding the best format of decision aids and the best graphic presentation in them of data on harms and benefits of treatment options remained unresolved.

The study by Holbrook and coauthors ${ }^{1}$ provides reassurance that methods in current use are equally good in helping patients arrive at informed decisions and that these benefits occur regardless of patient age or education level. The authors also noted a labelling effect (changes in preference based on knowing the name of the treatment), although confounding cannot be ruled out because labelling of the treatment options was not randomized. Labelling effects have been observed previously among patients and clinicians. ${ }^{7}$ For example, the attractiveness of surgery relative to radiation therapy for lung cancer was found to be greater when treatment options were identified than when they were not. ${ }^{7}$ Labelling may bring to mind other attributes of the processes involved, which are not evident in an unidentified description focusing solely on outcomes. In the case of anticoagulant therapy, patients with initial concerns about adding another drug may feel more reassured once they know it is acetylsalicylic acid, a familiar, inexpensive, accessible

\section{Key points about patient decision aids}

- Patient decision aids prepare patients to discuss "grey zone" treatment options and to clarify which benefits and harms matter most to them

- They differ from educational aids by not only providing option information but also tailoring it to the patient's clinical risk profile and guiding patients to express their personal values

- They promote informed values-based decisions and improve patient participation and comfort levels in decision-making

- They prevent the overuse of "grey zone" options that informed patients do not value (e.g., aggressive discretionary surgery such as hysterectomy, prostatectomy and back surgery)

- They will be used routinely at the point of clinical care only if they are made easy for clinicians to use and become something patients expect as part of high-quality care 
therapy. The international standards for patient decision aids maintain that information should be provided not only on outcomes of options but also on the processes involved. ${ }^{6}$

Although methodological studies will help us build better patient decision aids, we should not lose sight of the great gains that can be made with the adoption of existing ones. Randomized trials involving patients making real treatment or screening decisions have shown that standard counselling is inadequate: patients' knowledge was poor, their risk perceptions were unrealistic, and there was a mismatch between the option chosen and the benefits and harms that mattered most to them. ${ }^{3}$ Patient decision aids have consistently superior effects on these indicators of decision quality. They also help undecided patients decide, and they improve participation and comfort levels in decision-making.

Moreover, patient decision aids prevent the overuse of "grey zone" options that informed patients do not value, and consequently some decision aids are cost-neutral or costeffective. For example, decision aids have been found to reduce the rates of aggressive discretionary surgery (e.g., hysterectomy, prostatectomy, mastectomy, discectomy and coronary artery bypass graft surgery) by $25 \%$ and the rate of prostate-specific antigen testing by $20 \%$. It should be noted that decision aids do not always result in lower procedure rates. In cases of underuse, they may increase utilization; for example, rates of prostatectomy for benign prostatic hypertrophy increased in a setting where general practitioners' surgical referrals were low owing to a shortage of urologists. ${ }^{3}$

It is time to ensure that the uptake of "grey zone" options is consistent with the distribution of informed patients' preferences. By preventing overuse of options that informed patients do not value, resources can be reallocated to options that are currently underused. Patient decision aids may also be useful tools in managing the anticipated increase in wait lists for surgical consultations as the elderly population grows. A combination of standardized screening for surgical eligibility plus patient decision aids may make better use of surgeons' time so that they see patients who need and want discretionary surgery.

The routine use of patient decision aids at the point of clinical care will not happen unless decision aids are made easy for clinicians to use and become something patients expect as part of high-quality care ${ }^{8}$ We need to make patient decision aids a mandatory part of the informed consent process for "grey zone" decisions. Access to decision aids is improving (the provincial governments of British Columbia and
Saskatchewan have I30 patient decision aids on their consumer information Web sites), but it is not uniform across the country. Possible implementation strategies include "information prescriptions" (the provision of timely evidencebased health information to patients that is targeted to meet their specific needs and that supports sound decision-making [www.informationtherapy.org]) and "preference laboratories" (places where patients can view decision aids and answer a few key questions about their knowledge, values and preferences). An automated report can be sent back to the clinician to close the loop on decision-making with the patient. ${ }^{5}$ We also need to accelerate professional training in shared decision-making. ${ }^{8}$ Finally, new accreditation and reimbursement strategies that reward clinicians and other health care providers for the quality of "grey zone" decisions would accelerate the adoption of patient decision aids, with resulting benefits to patients, practitioners and the health care system.

Annette O'Connor is Tier I Canada Research Chair in Health Care Consumer Decision Support; Professor in the School of Nursing and the Department of Epidemiology and Community Medicine, University of Ottawa; and Senior Scientist, Clinical Epidemiology, Ottawa Health Research Institute, Ottawa, Ont.

Competing interests: None declared.

\section{REFERENCES}

I. Holbrook A, Labiris R, Goldsmith $\mathrm{CH}$, et al. Influence of decision aids on patient preferences for anticoagulant therapy: a randomized trial. $C M A J$ 2007;176:1583-7.

2. Thomson R, Parkin D, Eccles M, et al. Decision analysis and guidelines for anticoagulant therapy to prevent strokes in patients with atrial fibrillation. Lancet 2000;355:956-62.

3. O'Connor AM, Stacey D, Entwistle V, et al. Decision aids for people facing health treatment or screening decisions. Cochrane Database Syst Rev 2003;(2):CDoor43I.

4. McAlister FA, Man-Son-Hing M, Straus SE, et al. Impact of a patient decision aid on care among patients with nonvalvular atrial fibrillation: a cluster randomized trial. CMAJ 2005; I73:496-50I.

5. O'Connor AM, Wennberg JE, Légaré $\mathrm{F}$, et al. Towards the tipping point: accelerating the diffusion of decision aids and informed patient choice as a standard of practice. Health Aff. In press.

6. Elwyn G, O'Connor A, Stacey D, et al; International Patient Decision Aids Standards (IPDAS) Collaboration. Developing a quality criteria framework for patient decision aids: online international Delphi consensus process. BMJ 2006;333:4I7.

7. McNeil BJ, Pauker SG, Sox HC, et al. On the elicitation of preferences for alternatives therapies. N Engl J Med I982;306:I259-62.

8. Légaré F, Stacey D, Forest PG. Shared decision making in Canada: update, challenges and where next! $Z$ Arztl Fortbild Qual Gesundh. In press.

Correspondence to: Dr. Annette M. O'Connor, Clinical Epidemiology Program, Ottawa Health Research Institute, Rm. C-402, 1053 Carling Ave., Ottawa ON $\mathrm{KIY}_{4}$ Eg; aoconnor@ohri.ca 